\title{
Adaptive Case Management Framework to Develop Case-based Emergency Response System
}

\author{
Abobakr Y. Shahrah \\ Department of Software Engineering \\ King Saud University \\ Riyadh, Saudi Arabia
}

\author{
Majed A. Al-Mashari \\ Department of Information Systems \\ King Saud University \\ Riyadh, Saudi Arabia
}

\begin{abstract}
Emergency response to crisis, disaster, or catastrophe incidents is a clear example of knowledge-intensive and collaboration-heavy process facing all public safety-related organizations. Software systems to support emergency response have existed for decades. However, the limitations of these systems and their development approaches are still significant in terms of flexibility and dynamicity. With the emergence of Adaptive Case Management (ACM) as a new software development approach to support knowledge work and the empower knowledge worker, the authors found that ACM is a promising approach that can be extended to support emergency response especially in large-scale situations. This research aims to study how ACM can be leveraged to design and implement case-based emergency response systems (ERSs). In particular, the authors propose a domain-specific and vendor-neutral Case Management Framework (CMF) that incorporates the essential capabilities to support the ERSs. As a proof-of-concept, the authors support the proposed CMF by a case-based ERS prototype. Finally, the authors conclude that ACM has a great potential to enhance the effectiveness and efficiency of ERSs. This work can be considered as an attempt to advocate the adaptation of ACM in such context.
\end{abstract}

Keywords-Adaptive Case Management; Case Handling; Case Management; Emergency Response System

\section{INTRODUCTION}

Presently, there is a strong demand for more effective and efficient ways to respond to small or large-scale emergency incidents. This demand requires cohesive coordination among all emergency response stakeholders especially in the case of crises and disasters events [1]. With the advent of the Smart City vision that can be realized through various advanced technologies - e.g. internet-of-things - public safety personnel and residents expect smarter ways to manage emergency response with less effort and costs. Furthermore, the availability and affordability of sophisticated technologiese.g. pervasive sensors, multimedia surveillance, mobile computing, data analytics, social media, and cloud computing-make it quite possible to improve the effectiveness and efficiency of emergency response process [2]. Nevertheless, there are still major limitations associated with the traditional software development methodologies for supporting emergency response systems (ERSs) - e.g. Business Process Management (BPM) and Workflow technologies [3][4]. These approaches are too rigid and require considerable design and deployment cycles to enable missioncritical capabilities to deal with new or changing requirements.
This primarily returns to the unpredictability, unrepeatability, and complexity nature of the emergency response process, which is time-critical, knowledge-intensive, unstructured, very dynamic, and very complex to be completely predetermined [59]. Exceptions are norms in a crisis [10], and emergencies in most of the cases are not "routine" because each emergency can present unusual elements [11]. Therefore, ERSs must be scalable, flexible, and adaptable enough to enable a collaborative response approach, empower the coordination of engaged domain experts, and accommodate unexpected requirements - e.g. emergent course of actions - on the fly (run-time) [7, 12-15]. However, designing a software system to address an unstructured and adaptive process style is challenging $[4,16,17]$. In addition, there is still an imperative requirement for further domain-specific research and development in the BPM-related methods and tools to effectively support various use case applications such as ERSs [4, 18-20].

With the latest improvements in BPM research and practice in recent years, Adaptive Case Management (ACM) has been introduced as a promising paradigm shift to support knowledge-intensive processes (KIPs) - also known as knowledge work - in various application domains, such as healthcare, social services, legal services, insurance services, and many others. [16, 21-29]. ACM is a goal-and data-driven approach built on the traditional case management concepts that promote a collaborative and ad-hoc work to resolve cases and achieve certain goals. In fact, an emergency incident can be seen and treated as a case using the case management approach. Furthermore, emergency management (incident management) is one of the most appropriate application area for the ACM because it is a knowledge-intensive work process $[16,21,30-33]$. ACM offers the most flexibility and adaptability ever sought by emergency responders to deal with large-scale crises, disasters, or catastrophes. However, adopting $\mathrm{ACM}$ is not an easy or straightforward endeavor especially for complicated domain-specific applications, such as ERSs. ACM is still an emerging trend with many arguments on its capabilities, implementations, and challenges. Consequently, there is an open challenging research arena to deeply study what is exactly ACM and how it can support ERSs.

This research aims to investigate how ACM's concepts and technologies can be utilized to design and implement casebased ERSs. Specifically, the authors propose a domainspecific and vendor-neutral Case Management Framework (CMF) and describe its characteristics and capabilities to 
support ERSs. Furthermore, the authors demonstrate a casebased ERS prototype on a leading commercial CMF. This work can be a starting point to guide the design and development of case-based ERSs as well as an attempt to scope and formulate the ACM functionalities and capabilities in such context.

The rest of this paper is organized as follows: Section II is a summary of related works in BPM to support ERSs. Section III specifies the characteristics and capabilities of case management platform to support ERSs. Section IV describes the proposed CMF and its essential components to support the ERSs. Section V outlines the development methodology that should be considered to build effective case-based ERSs. Section VI presents a prototype demonstration of a case-based ERS. Finally, Section VII summarizes the key findings of this research and indicates possible future work.

\section{RELATED WORK}

The BPM and Workflow technologies are very useful and promising in developing the ERSs and have been discussed for several years [4, 34, 35]. Hofmann et al. [4] conducted a structured and comprehensive literature review to identify and discuss the BPM-related approaches-covering 98 scientific contributions from 1999 to Oct 2013 - in supporting disaster response management (DRM). They also consolidated a catalog of general requirements and research gaps for designing effective process-oriented DRM approaches. Wang et al. [36] presented a formal and intuitive approach for the modeling and analysis of workflows to support the flexibility of the incident command systems (ICSs).

The adaptation in BPM to support emergency response process is an emerging research direction to support incident management [18, 35]. De Leoni [37] described an adaptive process management system (PMS) called "SmartPM", which supports automatic adaptation techniques to cope with highly dynamic scenarios, such as emergency management. Catarci et al $[38,39]$ described the main results of a European project called WORKPAD, a two-level architecture (front-end and back-end) and used a user-centered design methodology that supports rescue operators in disaster response based on process management and geo-collaboration. Wagenknecht and Rueppel [40] presented a process-centric approach to support flood response management by using formal process models and adapting process management methods. They also suggested further research to achieve more flexibility in process support by focusing on dynamic changes of running processes.

Hofmann et al. [41] presented a (semi-)automated method called "DRP-ADAPT" to analyze disaster response process (DRP) models by considering place-related conflicts and their resolutions. However, this method is still based on some assumptions and limitations that might be irrelevant in practical scenarios. In addition, Hofmann et al. [42] extended the DRPADAPT by using the Precedence Diagram Method (PDM) to generate a list of valid execution traces with time consideration ranking.

Kushnareva et al. [5, 43] examined an activity-oriented paradigm of a flood management process implemented as a part of a smart city solution called "COS Operation Center"
(COSOC) and compared it with an alternative state-oriented and statecharts paradigm. Finally, they concluded that stateoriented modeling with statecharts has a great potential for modeling a crisis management process. Furthermore, Kushnareva et al [44] combined MAP (goal-oriented) and statecharts (state-oriented) formalisms to model and align the strategic and operational levels of crisis management as an intension-driven approach to a recommendation system of COSOC.

Most of the related works presented in this section (except the state-oriented research, which is more related to ACM) are based on the traditional BPM and Workflow technologies. These technologies are too restrictive and not flexible enough to support the knowledge work of emergency response, which is non-repeated, unpredictable, emergent, and requires a robustness in the face of varying conditions [29, 45-50]. BPM is a control-driven and an activity-centric approach to coordinate the control flow of the process activities based on a pre-specified process model. In contrast, ACM is a goal-driven and a data-centric approach to empower the knowledge worker to perform knowledge work (e.g. emergency response) with the help of a guiding template and adaptation capabilities. Despite the significant efforts in the last decade to make process-aware information systems (PAISs) more flexible in front of required process variability, looseness, adaptation, and evolution, case handling-i.e. ACM-is a paradigm shift to support knowledge-intensive work and enable user-and data-driven processes [51].

Cummins [52] described the ACM as the next generation BPM. ACM is using the principle of "design for people, build for change", which is a more lean approach than the structured and predicable approaches often automated with BPM suites [33]. ACM enables both the planning at design-time ("doingby-design") and planning at run-time ("design-by-doing") [25, 26]. ACM has both the ability of "flexibility-to-use" and "flexibility-to-change" to support knowledge work, whereas BPM and BPM 2.0 failed to provide support in this direction [53]. ACM typically uses functional languages (declarative) to enable dynamicity at the execution time instead of procedural languages (imperative) usually used in BPM [26, 54-57]. Some researchers discussed the strengths and weaknesses of BPM and ACM, and suggested a combination of both capabilities to overcome some inherited limitations when only considering one over the other [16, 58-60].

\section{CAPABILITIES OF ACM PLATFORM}

This section briefly highlights the common capabilities that mainly distinguish the ACM platforms from other approaches, such as traditional BPM platforms (also known as BPMS). These capabilities are very important to be considered when evaluating or selecting the appropriate ACM platform to develop and maintain the case-based ERSs.

Swenson [21] classified the ACM systems (platforms) capabilities into three levels: required in all ACM systems (level 1), common extensions in ACM systems (level 2), and advanced extensions for ACM systems (level 3). Level 1 includes the essential capabilities that a typical ACM system must support, such as team, folder, goals, history, security, communication, adaptation, and reporting capabilities. Level 2 
consists of some additional capabilities that can extend the ACM system, such as business entities, data interchange, business rules, resource state model, granular access control, sensors and triggers, and conformance guiding. Finally, Level 3 describes the advanced capabilities that can be considered to enhance the ACM system, such as process mining, social mining, federated case folders, ontology or taxonomy, and resource sharing.

Hill et al. [16] identified nine critical capabilities for evaluating commercial BPM-platform-based CMFs. These capabilities include adaptive case handling, content interaction, runtime collaboration, integration and interoperability, data capture and output management, out-of-the-box components, intelligent upgrades, role-based user experience, and roleappropriate analytics. The importance of each capability relies on the application use case. Gartner also provided a weighting for the nine critical capabilities based on four use cases: investigative cases, incident management, service request, and process-to-decision.

\section{PROPOSED ACM FRAMEWORK}

Developing ERSs is very complicated and requires special considerations for guiding design principles and concepts [10, 61-64]. These design principles and concepts consider the most important issues in emergency response requirements. In addition, frameworks and best practices provide an effective guidance in developing ERSs [64]. In this framework, the authors focus on the data and process technologies to enable effective and efficient case handling and decision-making.

In fact, there are many debates on what must be included in the ACM supporting platforms in terms of components and capabilities. The reason of these debates, in general, relies on the unavailability of a standardized framework and definition for a typical ACM. ACM industry vendors often look at the ACM from disparate perspectives based on their platforms' capabilities and technology offerings. The diversity of these perspectives has, unfortunately, increased the doubts of what is the ACM and what it must include.

The ACM market offers many generic case management frameworks (CMFs) that can serve a variety of domains' applications [26]. The problem with such offerings comes from the fact that a one-size-fits-all solution does not effectively work to support all various use cases in different domains. Therefore, there is a strong call from academic research for BPM and ACM domain-specific frameworks and systems [4, 18-20]. In addition, the ACM industry trend is to provide ready-made solution templates suitable for particular domain requirements to have a jump-start, reduce the time-to-market, and leverage pertained best practices.

Therefore, the authors propose a domain-specific and vendor-neutral CMF as illustrated in Fig. 1. This CMF can represent a high-level reference model for a ACM platform to effectively support ERS. The proposed CMF is composed of several components that are briefly described in the following subsections.

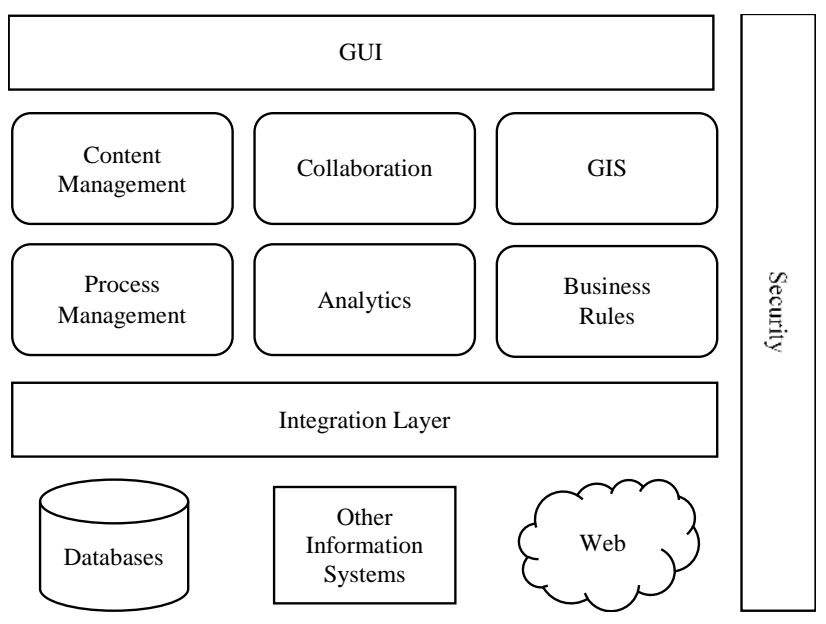

Fig. 1. Proposed adaptive case management framework for emergency response system

\section{A. Graphical User Interface (GUI)}

Graphical User Interface (GUI) is the medium for end users (e.g. emergency responders) to interact with the ERS. This includes all the input forms and output reports usually accessed by emergency-related personnel during or after the emergency incident. The GUI component has to be userfriendly and intuitive to support tired-dirty-hungry responders who operate under extreme stress. The GUI has also to combine and effectively display both graphical (e.g. map) and textual (e.g. incident detail) incident-related data in an appropriate manner that is suitable for different devices (e.g. desktop, laptop, notebook, and smartphones).

The GUI should be a web-based that is able to support multiple views based on various user requirements and authorization permissions. More importantly, the GUI has to be configurable with less hard-coding efforts to build or customize the system's look-and-feel, entry forms, or output reports.

\section{B. Content Management}

Data are the heart of ERS that Content Management is supporting an essential ACM component. Emergency situational awareness totally relies on the availability and accessibility of incident-related data, which include structured (e.g. rational databases) or unstructured (e.g. social media) contents. The structured data contain all of the incident's details (e.g. type, date and time, and location), responding units' details (e.g. type, name, numbers, and status), and responding activities (e.g. standard operating procedures, tasks assignments, and tasks status).

The unstructured data can include free-text, images, audios, videos that originated from social media or other sources (e.g. multimedia surveillance systems). The management of this complex information is very challenging, considering the information overload in the stressed and extreme events, as well as the privacy and security concerns pertaining to the incident, such as victims, responders, and places. 
Consequently, the Content Management component has to be capable and flexible to store, process, retrieve, and archive all kinds of incident-related data in an organized and structured manner-i.e. case folder. Furthermore, given that the emergency response is data-driven (situational awareness), the Content Management component has to be capable to drive and automatically initiate the response operations (tasks) based on content arrival or modification.

\section{Process Management}

Emergency response involves intensive coordination efforts to support cross-organizational personnel coming from multiple agencies (e.g. government, private sector, and nongovernmental organizations) and different levels (e.g. local, state, and federal), especially in the situation where the number of emergency responders exceeds the local capacity, and further state or federal assistance is requested. In fact, each emergency responding discipline (e.g. police, fire, and EMS) has to conform with its own emergency operations plan (EOPs), standard operating procedures (SOPs), and internal or external policies or regulations. Although there are many standard responding activities plans already in place before any incident occurrence, each incident case can present unique, emergent, and unpredictable challenges.

Therefore, traditional process styles (e.g. BPM and Workflow technologies) are inadequate to effectively support the management of emergency response operations because they are based on explicitly predefined process models that are too rigid to be modified at the run-time. In contrast, the Process Management component of ACM has to be adaptive and flexible to support handling of ad-hoc response actions at the run-time beside the capability to integrate with the traditional process styles, which might be required to run specific process fragments. In addition, the Process Management component has to interact seamlessly with the Content Management component to create task or trigger process segments based on data arrival or modification.

\section{Collaboration}

Knowledge exchange and information sharing among decision makers, emergency responders, and domain experts are fundamental requirements to effectively manage the emergency incident. People involved in emergency incident management used to communicate and coordinate their responding activities by using the traditional wireless telecommunications systems (e.g. mobile radio systems). However, from several past experiences and lessons learned, these approaches seem to be ineffective and insufficient due to the infrastructure and compatibility issues especially when different responding agencies are engaged with their own different telecommunications systems (e.g. different radio frequencies).

With ACM, the Collaboration component should provide flexible and effective means for sharing and discussing any details of the emergency incident among all responding participants at the real-time, such as using emails, instant messages (IMs), social media, and many others. ACM has to ensure a centralized and governed platform for knowledge sharing that can easily be accessed, monitored, archived, and audited at any time.

\section{E. Analytics}

The analysis and reporting of emergency incident-related data are crucial to support decision-making during the incident management. Getting insights from the rich structured and unstructured incident contents are very helpful at the real-time to enhance the response operations, and later to review the case historical data for auditing or compliance checking. The Analytics component plays a major role in the ACM to gather, analyze, process, and summarize the actionable knowledge pertaining to the emergency incidents. The Analytics component has to support all kinds of reporting and decisionmaking during or after the incident through the utilization of business intelligence and data mining techniques.

One example is the enabling of emergency responders and decision makers to define and monitor the required key performance indicators (KPIs) to ensure the maximum utilization of resources and activities during the incident management. Another important aspect of the Analytics component is the capability to guide the management of the current incident based on the course of actions and lessons learned from the prior similar incidents.

\section{F. Geographic Information System}

Geographic Information System (GIS) is an essential part of any ERS to support decision-making and enable effective collaboration and information sharing. GIS helps all incident management personnel in analyzing and visualizing the incident-related data on multi-layered maps. This improves the situational awareness and facilitates the identification of all incident-related places and affected areas. For example, the emergency responders can use the GIS to effectively understand the scope of the incident, plan the logistics, deploy the resources, support the search and rescue operations, define the evacuation paths, and others.

Moreover, the people affected by the incident can use the GIS to locate the evacuation roads, sheltering places, dangerous areas, and so on. The generic commercial ACM platforms do not usually encompass the GIS by default. However, the domain-specific ACM has to be capable of seamless integration and interaction with GIS to effectively support the emergency response use case.

\section{G. Business Rules}

ERSs are similar with other business applications that are governed by domain rules, government regulations, or policy compliance. The Business Rules component supports the decision-making and regulatory compliance as well as ensures the consistency and accuracy of the work outcomes. Traditionally, Business Rules were embedded into the software logic, such as control flow in the Workflow technologies. However, this approach is inefficient because to cope with any business rules updates or changes, the specialized developers might have to do frequent changes to different places in the software internal code.

Presently, the best practice is to separate the software logic from the rules logic to simplify the complexity of rules management through a centralized business rules engine (e.g. definition and update) that can be handled by business analysts instead of specialized developers. The Business Rules can then 
be utilized by multiple software applications and only need a single update to be reflected into all applications. In emergency response context, for example, the Business Rules can be used to automatically prioritize the emergency incident based on its details (e.g. type, size, and complexity), create or assign response tasks, or trigger process fragments (e.g. SOPs).

\section{H. Integration Layer}

ERS is not a stand-alone system and has to be integrated with multiple internal or external systems and databases, such as surveillance systems, citizens' records, medical records, hazardous material (HazMat) guides, and buildings blueprints. The Integration Layer component has to support the integration requirements of ERS to any system of records. This includes the ability to incorporate all types of data such as data on movement (e.g. surveillance or social media feeds) or data on rest (e.g. stored database records).

ACM has to provide a broad set of standardized application programming interfaces (APIs) or mediation technologies (e.g. enterprise service bus) to facilitate the interfacing requirements of ERS to any system or database-including any incidentrelated content available on the Web via the Internet.

\section{Security}

Security is an imperative component for ACM to effectively and efficiently support the ERS - especially when connected to the Internet. ERS is a mission-critical system that has to be secured from any potential cyber-attacks or security threats. ERS also includes sensitive data that has to be protected from any disruption or unauthorized access. The Security component has to ensure the confidentiality, integrity, and availability (CIA) of all the incident-related data with the appropriate required level of authentication and authorization. Emergency responders and other public safety personnel usually come from multiple agencies and have different information needs during or after the incident for either decision-making or reporting purposes.

ACM has to provide the flexible means to manage and maintain the ERS access rights and privileges based on different system levels (e.g. system access, system pages, and incident details) and users' roles (e.g. Incident Commander, Operations Section Chief, and Public Information Officer). ACM has also to support multiple system views to be created or customized according to each system user's requirements and conforming to any agency or government policies and regulations.

\section{DEVELOPMENT METHODOLOGY}

The design and development of case-based ERS are not a straightforward endeavor. The complexity, dynamicity, and variation of ERS requirements complicate the system development because they cannot be stably and completely defined in advance. Therefore, the traditional waterfall development approaches are inappropriate to build an effective and efficient case-based ERS. Agile software development methodologies can play an important role in this domain to mitigate the risks of changing requirements.

Agile approaches enable the iterative and incremental features development with high engagement of the knowledge workers (e.g. emergency responders) to evaluate and validate the introduced set of required features (prototype). Therefore, the improvements can be gradually attained by going through multiple iterations - considering the changing requirements and case workers' feedback. Recently, the DevOps approach (development and operations) has been introduced in the software development domain to bridge the gap between development and operation teams. The DevOps approach considers the agile methodologies along with other practices (e.g. continuous delivery and continuous deployment) to enhance the software development lifecycle, decrease the number of deployment failures, and reduce the time-to-market. ACM platforms should support the adoption of DevOps' practices to facilitate the effective development of case-based ERSs.

\section{PROTOTYPE}

To evaluate the capabilities of ACM to support the ERS, the authors develop a case-based emergency response prototype by using a leading ACM commercial platform [16, 65] called IBM Case Manager 5.2.1. The IBM Case Manager is deployed as a virtual machine (VM) image on our private IBM Cloud platform for academic research and teaching.

The IBM Case Manager includes many capabilities to enable flexibility for both design-time and run-time that can support the unpredictability and unrepeatability nature of the emergency response process. The core components of the IBM Case Manager to design and process case-based systems include Case Manager Builder, Case Manager Client, Case Manager API, Case Manager administration client, Content Platform Engine, and FileNet Workplace XT [47].

\section{A. Design}

In the ACM approach, the first step in system development is often to design a basic template that covers the predictive and essential requirements of the targeted system, such as case design, task design, case documents, and business rules. Creating an ERS using the IBM Case Manager includes three levels: defining the system with Case Manager Builder, refining the system by using more advanced configuration, and customizing the system by using custom code [47]. Fig. 2 shows a sample screenshot of the ERS essential definitions that include: defining case properties, defining roles, defining inbaskets, defining document types, defining case types, defining views, defining business rules, and defining tasks. 


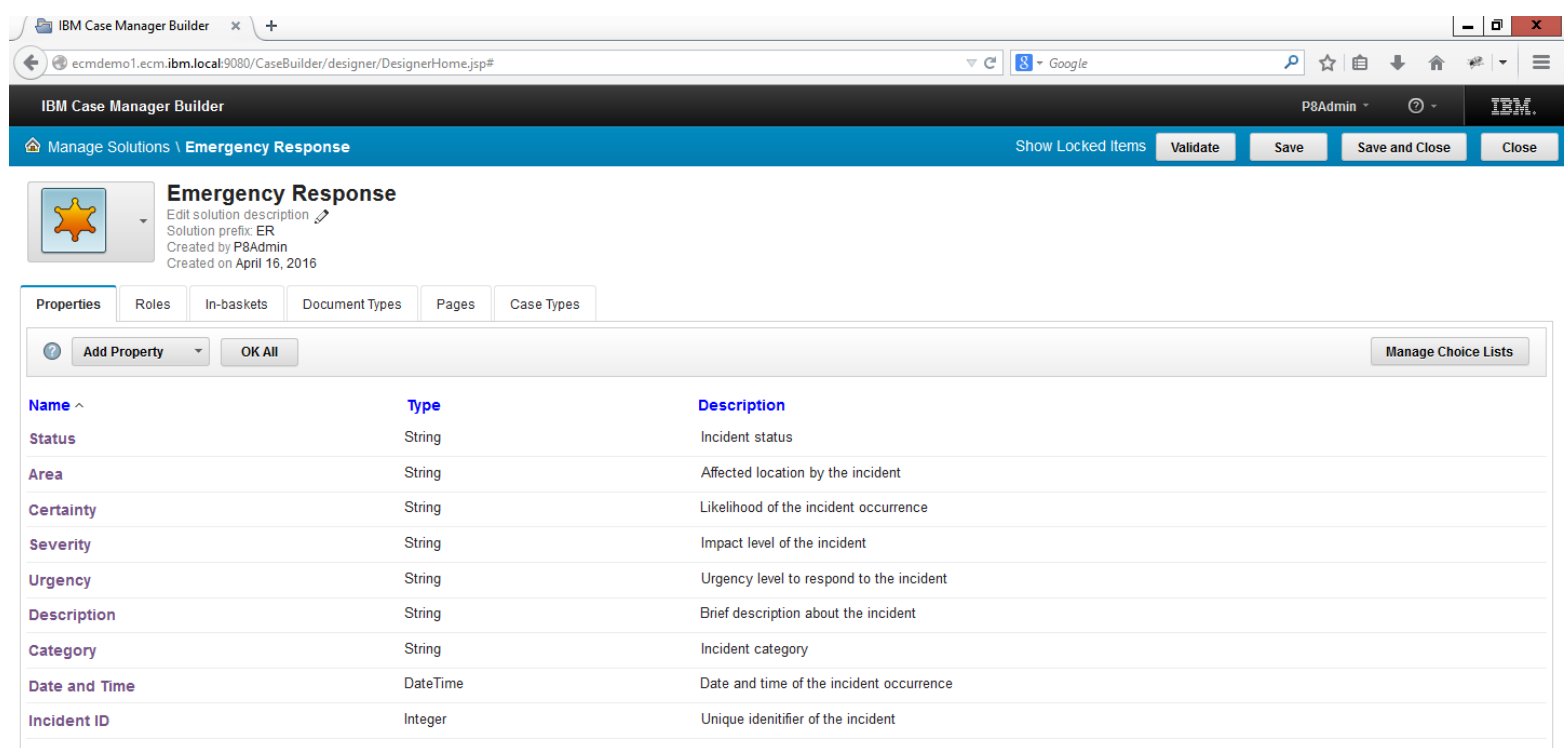

Fig. 2. Defining the ERS prototype with IBM Case Manager Builder

\section{B. Deployment and Testing}

The defined ERS prototype has been successfully deployed in the development environment. Fig. 3 shows the successful deployment status as a green rounded icon with a check mark in the IBM Case Manager Builder. The development environment enables the system analyst and end user to dynamically test and validate the developed functionalities, which can be achieved by creating a test case and checking the data and process flow as per required response actions defined in the design phase.

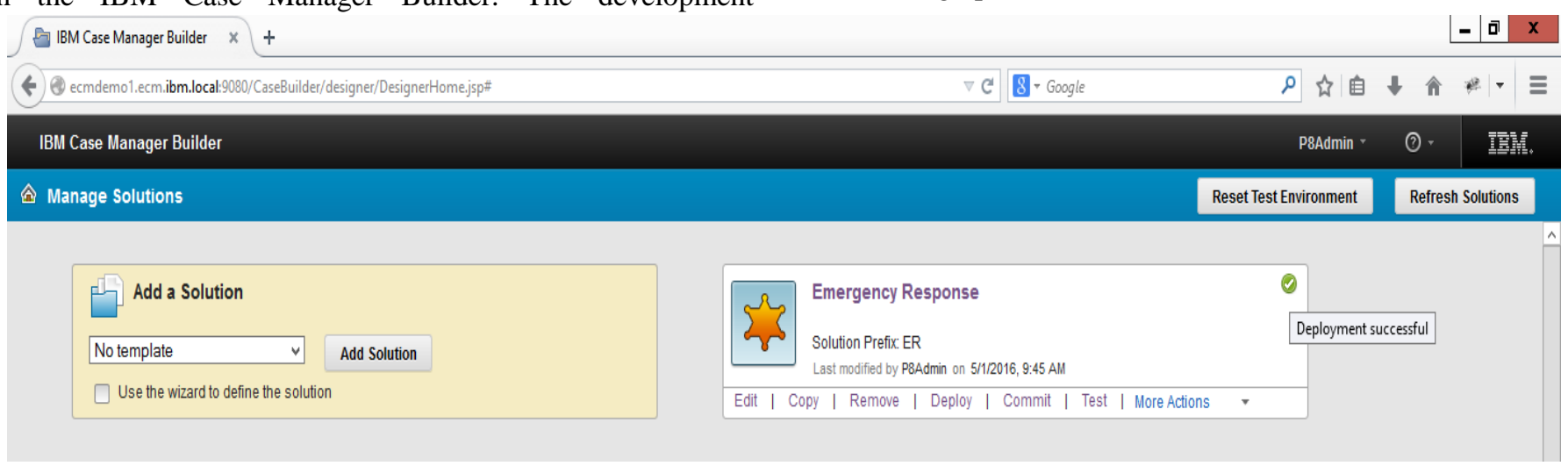

Fig. 3. ERS prototype deployment

Then, the deployed ERS prototype is tested by creating a sample incident case as shown in Fig. 4 (all entered values are fictitious for test purposes only). The creation of the incident case can be done manually or automatically. In manual creation, the end user (knowledge worker) enters the incident details manually based on the reported information of the incident via phone, papers, and others. On the other hand, in automatic creation, the incident details are entered automatically by fetching the data from other systems such as detection systems, computer-aided dispatch (CAD), and others. More details on the incident can also be accessed from other sources such as internal or external databases. 


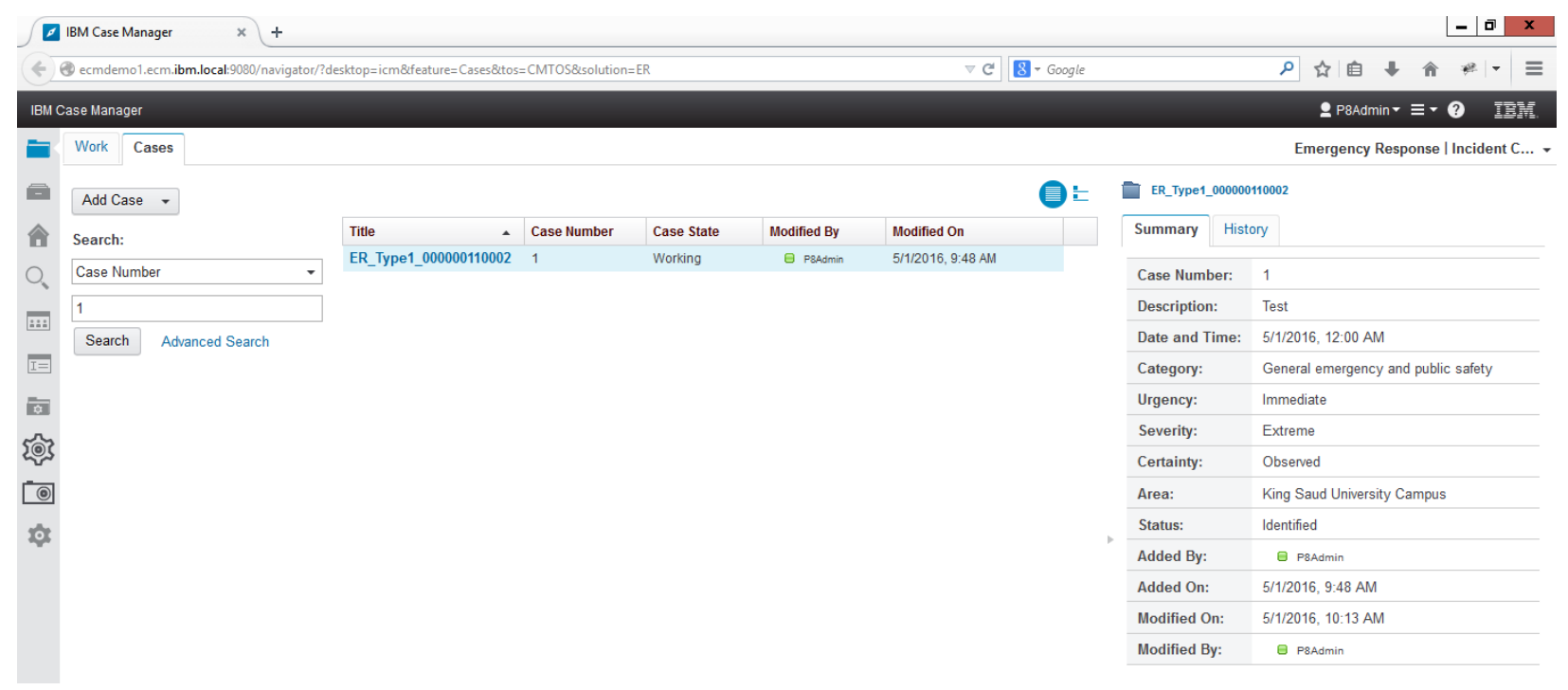

Fig. 4. An example of an incident case

As shown in Fig. 5, the ACM platform has automatically created a set of different tasks, and assigned them to different roles based on the defined template. These tasks appeared with three different statuses: Waiting (waiting for a precondition), Ready (manual task), and Started (automatically started). The active tasks are with the status Started and are automatically sent to the corresponding role in-basket that can only be accessed by the authorized user. The Waiting task is started when the precondition(s) is met. The Ready task can be started when manually initiated by the user (discretionary tasks).

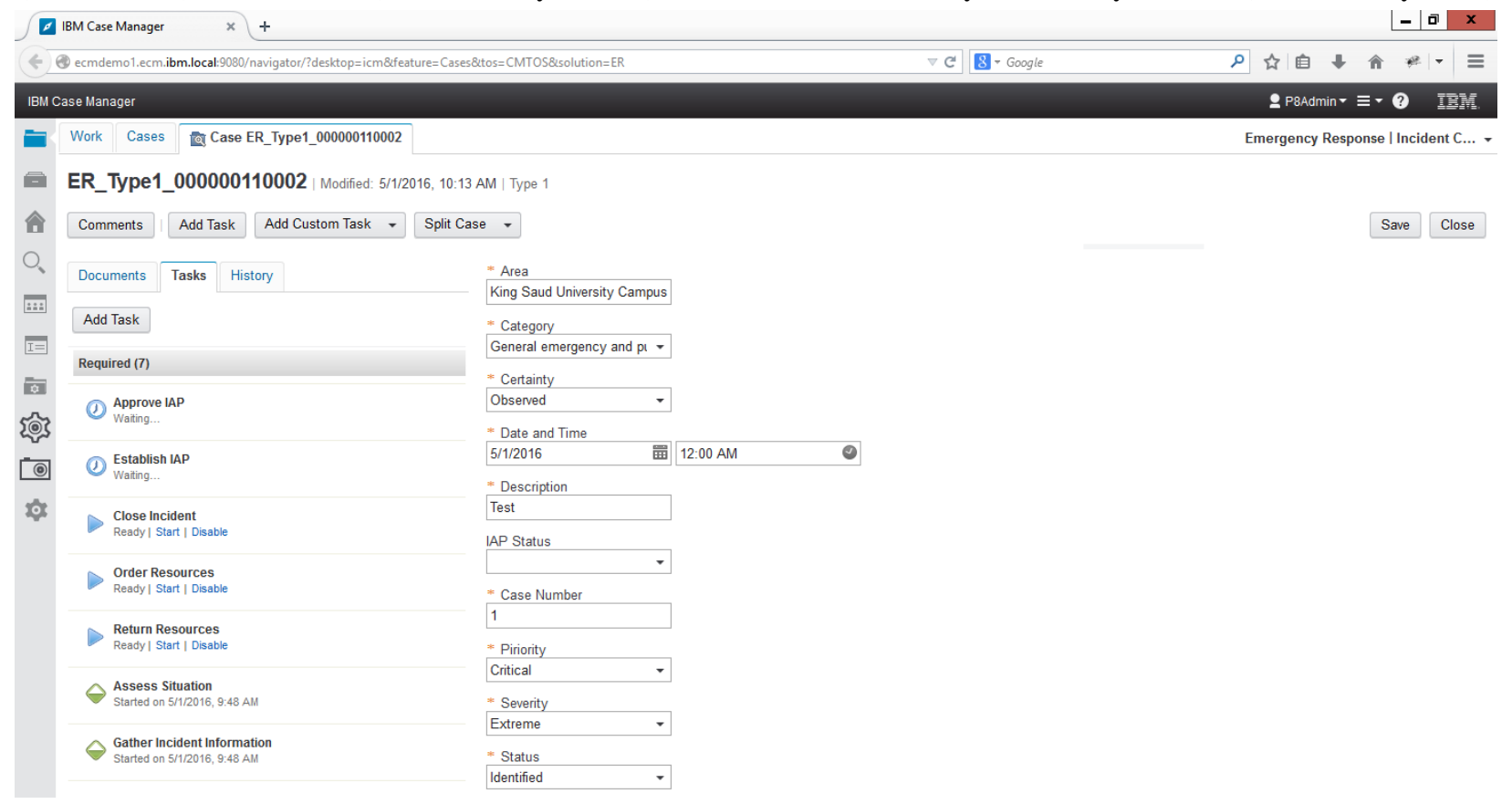

Fig. 5. Tasks automatically generated for the sample incident case

\section{CONCLUSION AND FUTURE WORK}

The response to large-scale emergency incident, such as crisis, disaster, or catastrophe, is a complicated process and poses a challenge to public safety personnel. Large-scale emergency incidents are unpredictable and unrepeatable, which make incident management and decision-making very challenging. These incidents often exceed the local capacity and require additional support from state or federal agencies, which implies intensive coordination and management of hundreds (or even thousands) of responders from diverse government, private sector, and non-governmental organizations.

In the past decades, many attempts have been carried out to design and develop sophisticated information systems to support emergency response utilizing the latest technological advances. Nonetheless, those efforts are still inadequate to effectively and efficiently empower the emergency knowledge workers who require a flexible, adaptable, and scalable system 
to handle large-scale emergency incidents. Most of the existing emergency response systems are built based on traditional software development approaches-e.g. Business Process Management and Workflow. These approaches are too rigid, require considerable time and effort to be customized or modified, and restrict the emergency responders from adapting to the dynamic and emergent requirements during the response operations.

With the introduction of Adaptive Case Management as an emergent knowledge work support approach, the authors found that there is great potential to extend the ACM concepts and technologies beyond the traditional use cases, such as healthcare, legal services, and insurance. ACM is a goal-and data-driven approach that can effectively and efficiently support the concept of management-by-objectives recommended in handling the emergency response. ACM can deal with all types of data and processes (structured and unstructured) and can also provide the emergency responders with the most flexible and adaptable mechanisms to define the course of action at the design-time (defined template) or even during the run-time (ad-hoc activities).

In this research, the authors proposed a domain-specific and vendor-neutral Case Management Framework that incorporates all essential capabilities to develop an effective and efficient case-based ERS. The proposed CMF consists of nine major components: Graphical User Interface, Content Management, Process Management, Collaboration, Analytics, Geographic Information System, Business Rules, Integration Layer, and Security. The authors outlined the required capabilities and characteristics of each component to support the ERS in accordance with the ACM concepts and principles. In addition, the authors indicated that the proposed CMF should be accompanied with an agile development methodology to facilitate a rapid system development and mitigate any associated risks.

In addition to the proposed CMF, the authors demonstrated a prototype on a leading commercial ACM platform (IBM Case Manager) to evaluate the basic capabilities necessary to design, develop, and deploy a case-based ERS. The authors concluded that ACM-with its advanced capabilities-has great potential to enhance the emergency response by significantly including situational awareness, response coordination, and decision-making.

Although the authors attempted to study, investigate, and evaluate how the ACM can support the ERS, this work still encompasses some limitations. The major limitation is the lack of real-life implementation because the authors performed this research study on a lab-setting with limited access to actual resources that are usually required in real-life situations. The authors admit that real-life implementation is more complicated, and usually includes technological and nontechnological challenges.

Finally, our future work will be focused on the real-life implementation considerations by involving subject matter experts (SMEs) to refine and discuss how the ACM can help in designing, developing, and implementing case-based ERS in the real-life context. Additionally, further research is required to investigate how ERS can benefit from data analytics capability in ACM to predict and enhance incident handling based on knowledge of similar previous incidents.

\section{REFERENCES}

[1] G. Nestler and A. Jackman, "21st century emergency management," in Smarter Cities, Thought Leadership White Paper, ed: IBM Corporation, 2014.

[2] N. R. Council, Improving disaster management: The role of it in mitigation, preparedness, response, and recovery. Washington, DC: The National Academies Press, 2007.

[3] G. Peinel and T. Rose, "Business processes and standard operating procedures: Two coins with similar sides," in Electronic government. vol. 8074, M. Wimmer, M. Janssen, and H. Scholl, Eds., ed: Springer Berlin Heidelberg, 2013, pp. 224-236.

[4] M. Hofmann, H. Betke, and S. Sackmann, "Process-oriented disaster response management: A structured literature review," Business Process Management Journal, vol. 21, pp. 966-987, 2015.

[5] E. Kushnareva, I. Rychkova, and B. Le Grand, "Modeling business processes for automated crisis management support: Lessons learned," in IEEE Ninth International Conference on Research Challenges in Information Science, Athens, Greece, 2015.

[6] B. Bennet, "Effective emergency management: A closer look at the incident command system," Professional Safety, vol. 56, pp. 28-37, 2011.

[7] D. Mendonça, "Decision support for improvisation in response to extreme events: Learning from the response to the 2001 world trade center attack," Decision Support Systems, vol. 43, pp. 952-967, 2007.

[8] C. Di Ciccio, A. Marrella, and A. Russo, "Knowledge-intensive processes: Characteristics, requirements and analysis of contemporary approaches," Journal on Data Semantics, vol. 4, pp. 29-57, 2014.

[9] S. Jul, "Who's really on first? A domain-level user, task and context analysis for response technology," in Proceedings of the 4th International Conference on Information Systems for Crisis Response and Management ISCRAM 2007, 2007, pp. 139-148.

[10] M. Turoff, M. Chumer, B. V. de Walle, and X. Yao, "The design of a dynamic emergency response management information system (dermis)," Journal of Information Technology Theory and Application (JITTA), vol. 5, p. 3, 2004.

[11] M. K. Lindell, "Emergency management," in Encyclopedia of natural hazards, P. T. Bobrowsky, Ed., ed Dordrecht: Springer Netherlands, 2013, pp. 263-271.

[12] M. Turoff, "Past and future emergency response information systems," Commun. ACM, vol. 45, pp. 29-32, 2002.

[13] U.S. Department of Homeland Security, "National response framework," Second Edition ed, 2013.

[14] M. Turoff, C. White, and L. Plotnick, "Dynamic emergency response management for large scale decision making in extreme hazardous events," in Supporting real time decision-making. vol. 13, F. Burstein, P. Brézillon, and A. Zaslavsky, Eds., ed: Springer US, 2011, pp. 181-202.

[15] B. Diirr, M. Borges, and D. Mendonça, "Putting plans on track in unforeseen situations," presented at the ISCRAM 2015 Conference Proceedings - 12th International Conference on Information Systems for Crisis Response and Management, Kristiansand, Norway, 2015.

[16] J. B. Hill, R. Dunie, and K. Chin, "Critical capabilities for bpmplatform-based case management frameworks," ed: Gartner, Inc., 2015.

[17] D. Schuerman, K. Schwarz, and a. B. Williams. (2014). Dynamic case management for dummies (Pega Special Edition ed.).

[18] M. Hofmann, S. Sackmann, and H. Betke, "Using workflow management systems to improve disaster response processes," in Advanced Information Networking and Applications Workshops (WAINA), 2013 27th International Conference on, 2013, pp. 261-266.

[19] H. Sem, S. Carlsen, and G. Coll, "On two approaches to acm," in Business process management workshops. vol. 132, M. La Rosa and P. Soffer, Eds., ed: Springer Berlin Heidelberg, 2013, pp. 12-23.

[20] H. Betke, "Structure and elements of disaster response processes - a general meta-model," presented at the ISCRAM 2015 Conference Proceedings - 12th International Conference on Information Systems for Crisis Response and Management, Kristiansand, Norway, 2015. 
[21] K. D. Swenson, "State of the art in case management," White paper ed: Fujitsu America, Inc., 2013.

[22] H. de Man, "Case management: A review of modeling approaches," BPTrends, January, vol. 2009, 2009.

[23] M. Marin, R. Hull, and R. Vaculín, "Data centric bpm and the emerging case management standard: A short survey," in Business Process Management Workshops, 2013, pp. 24-30.

[24] D. Miles, "Broadening the scope for advanced case management," ed: AIIM White Paper, 2013.

[25] Object Management Group (OMG), "Case management model and notation, version 1.0," ed, 2014.

[26] J. B. Hill, K. Chin, and R. Dunie, "Magic quadrant for bpm-platformbased case management frameworks," ed: Gartner, Inc., 2015.

[27] I. Cano, A. Alonso, C. Hernandez, F. Burgos, A. Barberan-Garcia, J. Roldan, et al., "An adaptive case management system to support integrated care services: Lessons learned from the nexes project," Journal of Biomedical Informatics, vol. 55, pp. 11-22, 2015.

[28] W. M. P. v. d. Aalst and P. J. S. Berens, "Beyond workflow management: Product-driven case handling," presented at the Proceedings of the 2001 International ACM SIGGROUP Conference on Supporting Group Work, Boulder, Colorado, USA, 2001.

[29] W. M. P. van der Aalst, M. Weske, and D. Grünbauer, "Case handling: A new paradigm for business process support," Data \& Knowledge Engineering, vol. 53, pp. 129-162, 2005.

[30] M. Kirsch-Pinheiro and I. Rychkova, "Dynamic context modeling for agile case management," in On the Move to Meaningful Internet Systems: OTM 2013 Workshops, 2013, pp. 144-154.

[31] N. Ward-Dutton, "The case for smarter case management," ed: MWD Advisors, 2013.

[32] IBM, "Advanced case management leadership guide," ed: IBM ECM, 2013.

[33] C. Le Clair and C. Moore. (2009, Dynamic case management-an old idea catches new fire. Forrester Research.

[34] D. Hagebölling and M. de Leoni, "Supporting emergency management through process-aware information systems," in Business process management workshops. vol. 17, D. Ardagna, M. Mecella, and J. Yang, Eds., ed: Springer Berlin Heidelberg, 2009, pp. 298-302.

[35] M. de Leoni, A. Marrella, and A. Russo, "Process-aware information systems for emergency management," in Towards a service-based internet. Servicewave 2010 workshops. vol. 6569, M. Cezon and Y. Wolfsthal, Eds., ed: Springer Berlin Heidelberg, 2011, pp. 50-58.

[36] J. Wang, D. Rosca, W. Tepfenhart, A. Milewski, and M. Stoute, "Dynamic workflow modeling and analysis in incident command systems," Systems, Man and Cybernetics, Part A: Systems and Humans, IEEE Transactions on, vol. 38, pp. 1041-1055, 2008.

[37] M. de Leoni, "Adaptive process management in highly dynamic and pervasive scenarios," presented at the Proceedings Fourth European Young Researchers Workshop on Service Oriented Computing, 2009.

[38] T. Catarci, M. d. Leoni, A. Marrella, M. Mecella, A. Russo, R. Steinmann, et al., "Workpad: Process management and geocollaboration help disaster response," International Journal of Information Systems for Crisis Response and Management (IJISCRAM), vol. 3, pp. 32-49, 2011.

[39] T. Catarci, M. de Leoni, A. Marrella, M. Mecella, B. Salvatore, G. Vetere, et al., "Pervasive software environments for supporting disaster responses," Internet Computing, IEEE, vol. 12, pp. 26-37, 2008.

[40] A. Wagenknecht and U. Rueppel, "Process driven emergency management platform for flood response integrating web gis technologies," International Journal of Civil \& Environmental Engineering, vol. 13, p. 1, 2013.

[41] M. Hofmann, H. Betke, and S. Sackmann, "Automated analysis and adaptation of disaster response processes with place-related restrictions," presented at the ISCRAM 2015 Conference Proceedings - 12th International Conference on Information Systems for Crisis Response and Management, 2015.

[42] M. Hofmann, S. Sackmann, and H. Betke, "Using precedence diagram method in process-oriented disaster response management," presented at the ISCRAM 2015 Conference Proceedings - 12th International
Conference on Information Systems for Crisis Response and Management, Kristiansand, Norway, 2015.

[43] E. Kushnareva, I. Rychkova, and B. Le Grand, "Modeling and animation of crisis management process with statecharts," in Perspectives in business informatics research. vol. 229, R. Matulevičius and M. Dumas, Eds., ed: Springer International Publishing, 2015, pp. 145-160.

[44] E. Kushnareva, I. Rychkova, R. Deneckére, and B. Le Grand, "Modeling crisis management process from goals to scenarios," in AdaptiveCM 2015-4th International Workshop on Adaptive Case Management and other non-workflow approaches to BPM, 2015.

[45] L. Fischer. (2010). Bpm and workflow handbook, spotlight on business intelligence.

[46] Forrester, "The next generation of knowledge worker processes will dominate enterprises," ed: Forrester Research, Inc., 2010.

[47] W.-D. Zhu, B. Benoit, B. Jackson, J. Liu, M. Marin, S. Meena, et al., Advanced case management with ibm case manager, Fourth ed.: IBM Redbooks, 2014.

[48] W. M. Van der Aalst, M. Weske, and D. Grünbauer, "Case handling: A new paradigm for business process support," Data \& Knowledge Engineering, vol. 53, pp. 129-162, 2005.

[49] K. Kaan, H. Reijers, and P. van der Molen, "Introducing case management: Opening workflow management's black box," in Business process management. vol. 4102, S. Dustdar, J. Fiadeiro, and A. Sheth, Eds., ed: Springer Berlin Heidelberg, 2006, pp. 358-367.

[50] A. Fink and S. Vogt, "Managing knowledge-intensive business processes by harnessing collective practical experience without codification," in S-bpm one - application studies and work in progress. vol. 422, C. Zehbold, Ed., ed: Springer International Publishing, 2014, pp. 107-121.

[51] M. Reichert and B. Weber, Enabling flexibility in process-aware information systems: Challenges, methods, technologies: Springer Science \& Business Media, 2012.

[52] F. Cummins, "Bpm meets soa: A new era in business design," in Handbook on business process management 1, J. vom Brocke and M. Rosemann, Eds., ed: Springer Berlin Heidelberg, 2015, pp. 531-555.

[53] C. Herrmann and M. Kurz, "Adaptive case management: Supporting knowledge intensive processes with it systems," in S-bpm one-learning by doing-doing by learning, ed: Springer, 2011, pp. 80-97.

[54] E. Morrison, A. Ghose, H. Dam, A. Menzies, and K. Khodaei, "Declarative service modeling through adaptive case management," in Handbook of research on demand-driven web services: Theory, technologies, and applications, Z. Sun, Ed., ed: IGI Global, 2014, p. 152.

[55] T. Hildebrandt, M. Marquard, R. Mukkamala, and T. Slaats, "Dynamic condition response graphs for trustworthy adaptive case management," in On the move to meaningful internet systems: Otm 2013 workshops. vol. 8186, Y. Demey and H. Panetto, Eds., ed: Springer Berlin Heidelberg, 2013, pp. 166-171.

[56] T. Slaats, R. Mukkamala, T. Hildebrandt, and M. Marquard, "Exformatics declarative case management workflows as dcr graphs," in Business process management. vol. 8094, F. Daniel, J. Wang, and B. Weber, Eds., ed: Springer Berlin Heidelberg, 2013, pp. 339-354.

[57] M. Marquard, M. Shahzad, and T. Slaats, "Web-based modelling and collaborative simulation of declarative processes," in Business process management. vol. 9253, H. R. Motahari-Nezhad, J. Recker, and M. Weidlich, Eds., ed: Springer International Publishing, 2015, pp. 209225.

[58] K. Traganos and P. Grefen, "Hybrid service compositions: When bpm meets dynamic case management," in Service oriented and cloud computing. vol. 9306, S. Dustdar, F. Leymann, and M. Villari, Eds., ed: Springer International Publishing, 2015, pp. 226-239.

[59] D. Auer, S. Hinterholzer, J. Kubovy, and J. Küng, "Business process management for knowledge work: Considerations on current needs, basic concepts and models," in Novel methods and technologies for enterprise information systems. vol. 8, F. Piazolo and M. Felderer, Eds., ed: Springer International Publishing, 2014, pp. 79-95.

[60] H. A. Reijers, J. H. M. Rigter, and W. M. P. van der Aalst, "The case handling case," International Journal of Cooperative Information Systems, vol. 12, pp. 365-391, 2003. 
[61] R. Chen, R. Sharman, H. R. Rao, and S. Upadhyaya, "Design principles of coordinated multi-incident emergency response systems," in Intelligence and security informatics, ed: Springer, 2005, pp. 81-98.

[62] R. Chen, R. Sharman, H. R. Rao, and S. Upadhyaya, "Design principles for critical incident response systems," Information Systems and EBusiness Management, vol. 5, pp. 201-227, 2007.
[63] J. K. Kim, R. Sharman, H. R. Rao, and S. Upadhyaya, "Efficiency of critical incident management systems: Instrument development and validation," Decision Support Systems, vol. 44, pp. 235-250, 2007.

[64] S. Shan, L. Wang, L. Li, and Y. Chen, "An emergency response decision support system framework for application in e-government," Information Technology and Management, vol. 13, pp. 411-427, 2012.

[65] C. L. Clair, A. Cullen, E. Cullen, and D. Lynch, "The forrester wave ${ }^{\mathrm{TM}}$ : Dynamic case management, q1 2016," 2016. 